ارزيابى اكوتيبهاى كاودانه از نظر تحمل به خشكى

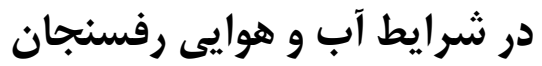

\begin{tabular}{|c|}
\hline 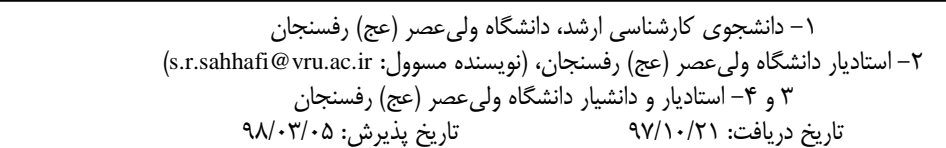 \\
\hline صفحه: \\
\hline
\end{tabular}

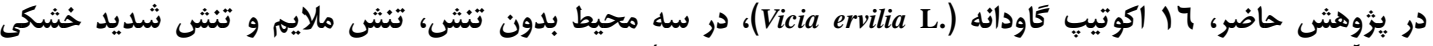

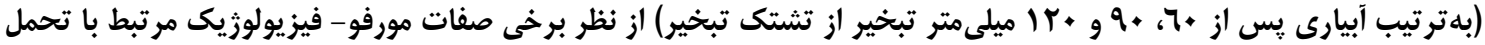

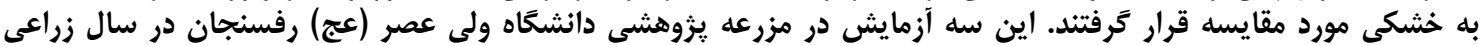

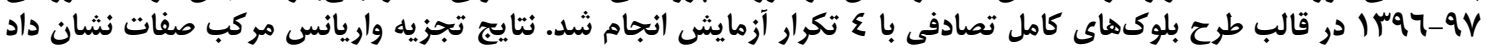

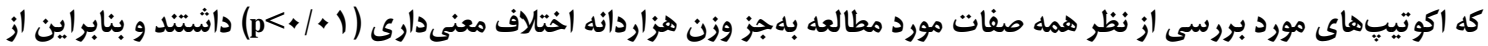

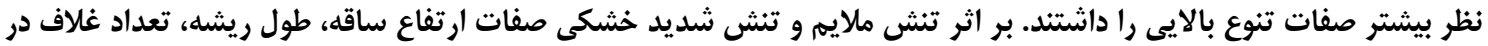

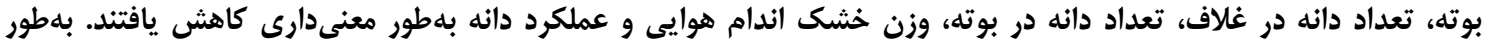

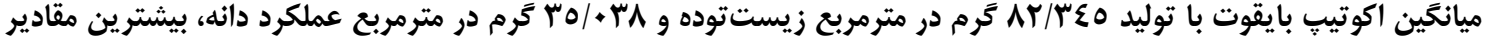

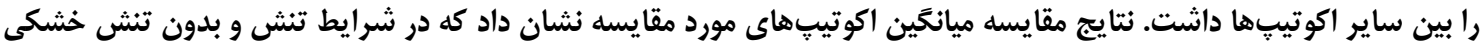

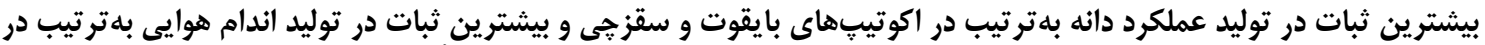

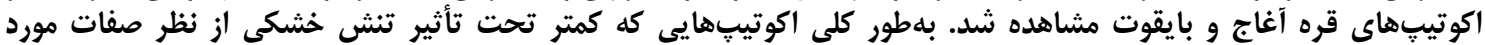

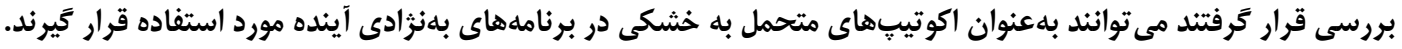

وازههاى كليدى: كَاودانه، تنش خشكى، مقاومت، عملكرد دانه

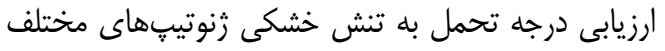

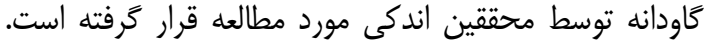

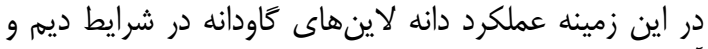

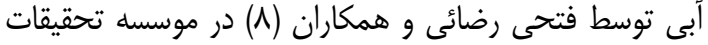

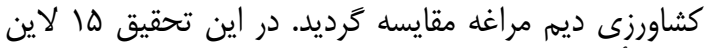

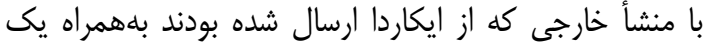

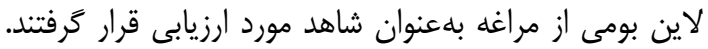

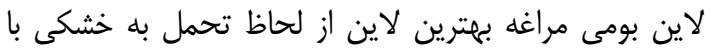

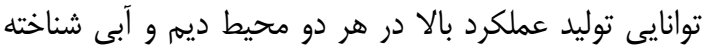

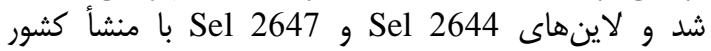

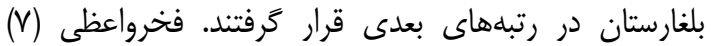

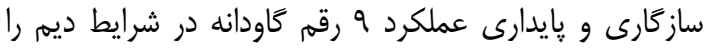

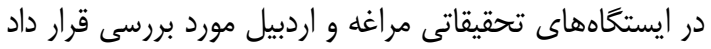

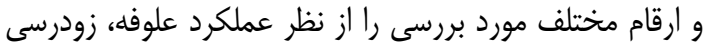

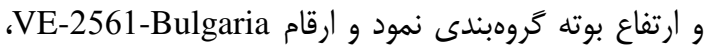

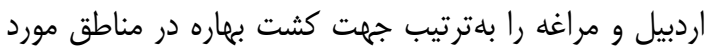

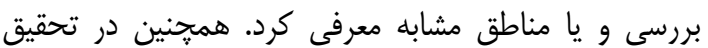

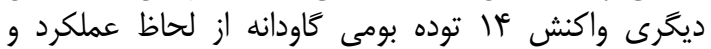

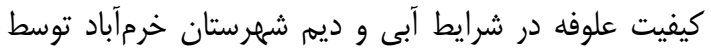

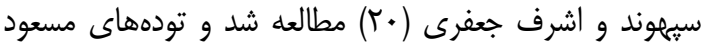

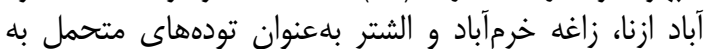

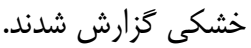

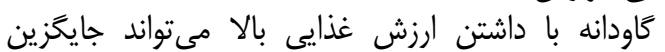

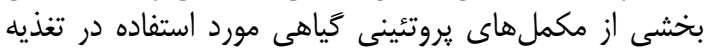

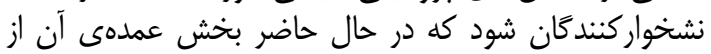

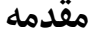

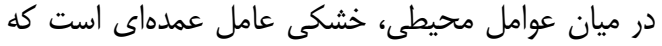

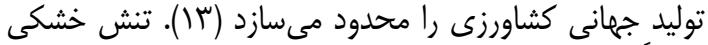

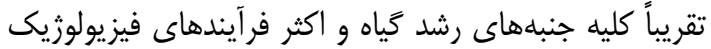

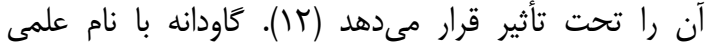

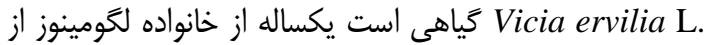
جنس ماشكها، زير تيره بروانهآسايان (Papilionaceae) و

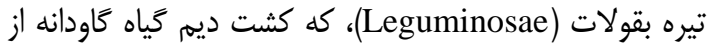

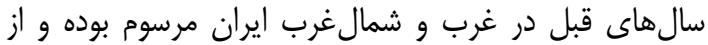

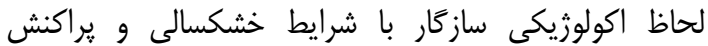

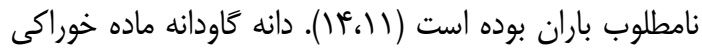

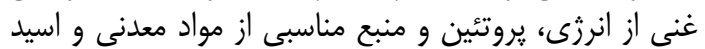

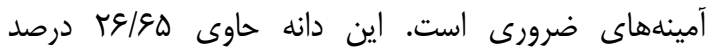

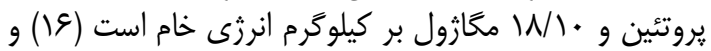

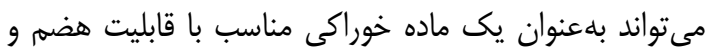

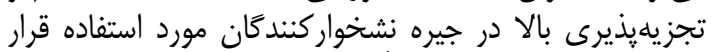

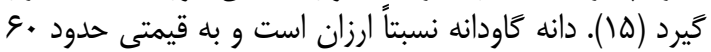

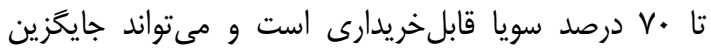

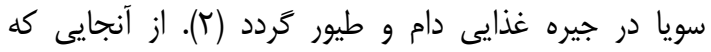

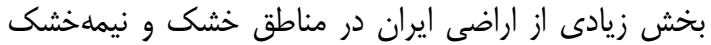

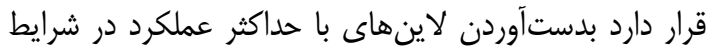

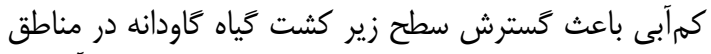

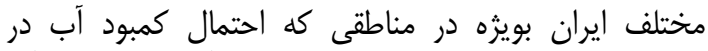
مراحل حساس رشدى بالا بوده و يا كمبود آب مانع كشت آن آن مىشود، خواهد شد. 
زراعى- مورفولوزيك صورت گرفت. مشخصات اكوتيڤهاى

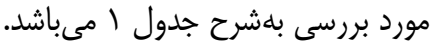

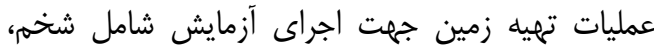

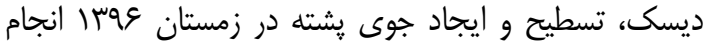

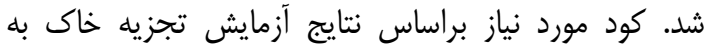

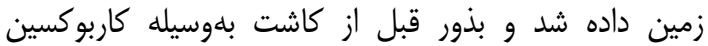

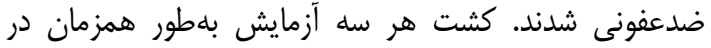

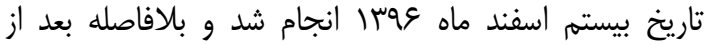

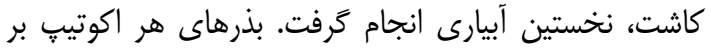

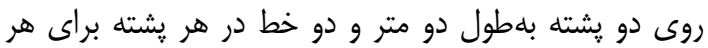

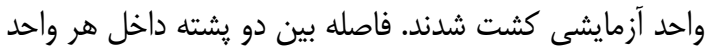

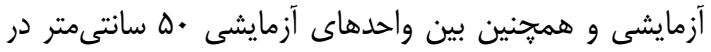

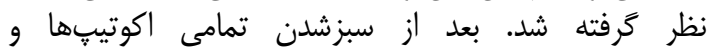

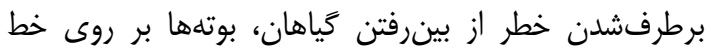

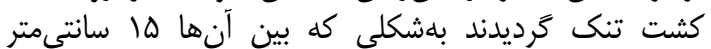

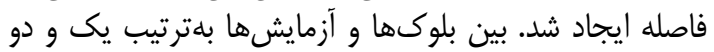

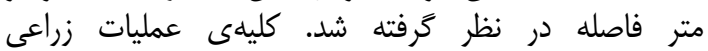

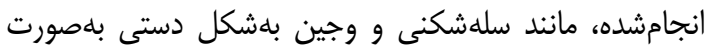

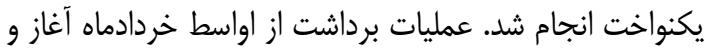

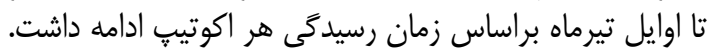

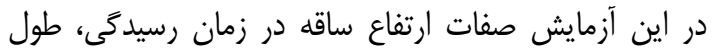

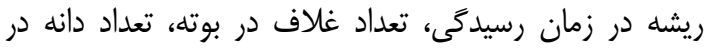

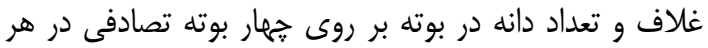

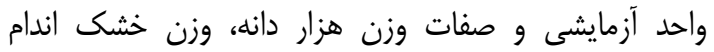

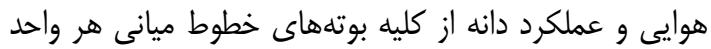

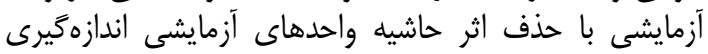

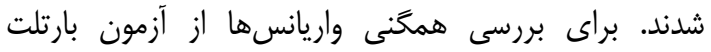

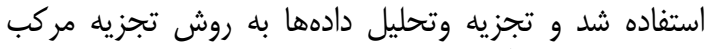
بوسيله نرمافزار آمارى 9.1 SAS

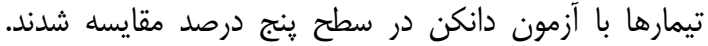

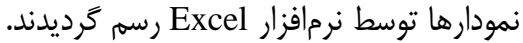

خارج كشور تأمين مىشود. باتوجه به شرايط آب و آب هوايى إيى

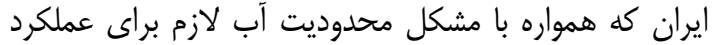

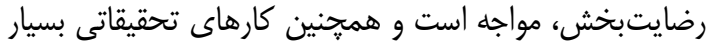

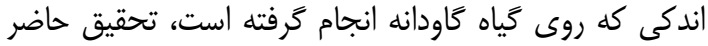

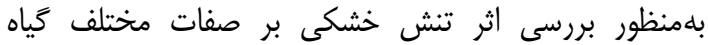

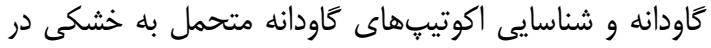
شرايط اقليمى شهرستان رفسنجان انجام كرفت.

\section{مواد و روشها}

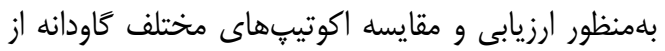

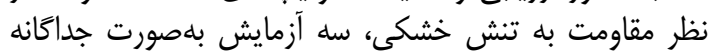

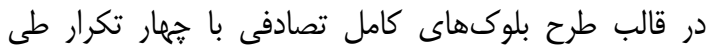

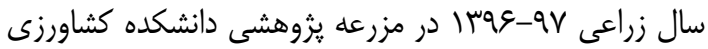

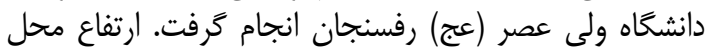

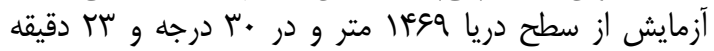

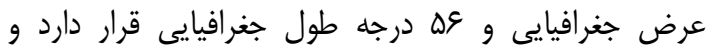

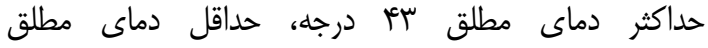

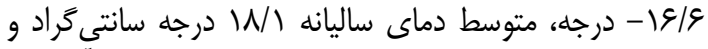

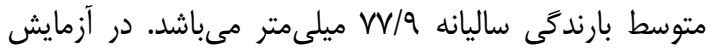

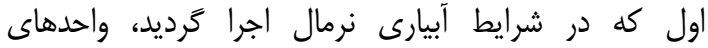

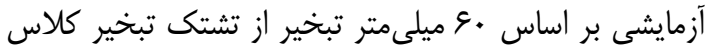

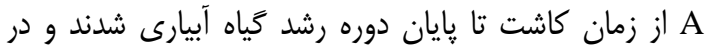

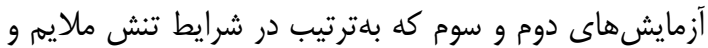

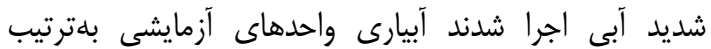

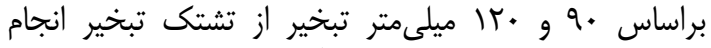

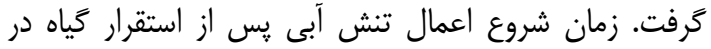

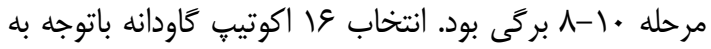

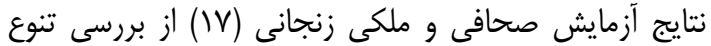

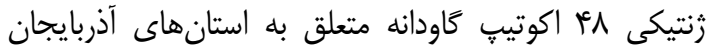

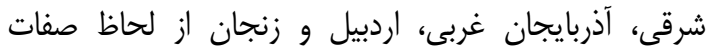

جدول 1- اكوتيڤهاى گاودانه مورداستفاده در آزمايش

Table 1. The list of studied bitter vetch ecotypes

\begin{tabular}{|c|c|c|c|c|c|}
\hline محل جمع آورى (استان-شهرستان-روستا) & نام توده & شماره & محل جمعآورى (استان-شهرستان-روستا) & 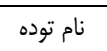 & شماره \\
\hline آذربايجان غربى-خوى -روستاى ميدان & ميدان & 9 & آذربايجان شرقى -اهر -روستاى بهل & بهل & 1 \\
\hline اردبيل-خلخال-روستاى آلهاشم & 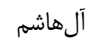 & 1. & آذربايجان شرقى-سراب-روستاى شربيان & 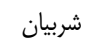 & r \\
\hline اردبيل-كرمى -روستاى قره آغاج & قره آغاج & 11 & آذربايجان شرقى-مراغه-روستاى حاجى كرد & 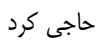 & r \\
\hline اردبيل-مشكين شهر-روستاى ساربانلار & ساربانلار & ir & آذربايجان شرقى-ملكان -روستاى بايقوت & 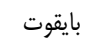 & c \\
\hline اردبيل -ممين-روستاى سقزيى & سقزجى & זו & آذربايجان شرقى-ميانه-روستاى شيخدرآباد & 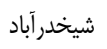 & $\Delta$ \\
\hline زنجان-ابهر-روستاى داش بلاغ & 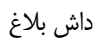 & 14 & آذربايجان شرقى -هريس-روستاى برازين & 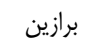 & 9 \\
\hline زنجان-زنجان-روستاى جومالو & 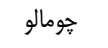 & 10 & آذربايجان غربى-حالداران-روستاى قورول & 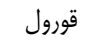 & 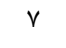 \\
\hline زنجان-زنجان-روستاى كلوجه & 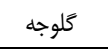 & 19 & آذربايجان غربى-خوى-روستاى الند & 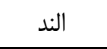 & $\wedge$ \\
\hline
\end{tabular}

وزن هزاردانه اختلاف معنىدارى با هم داشتند كه نشاندهنده

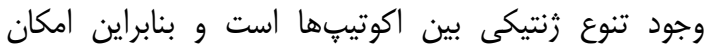

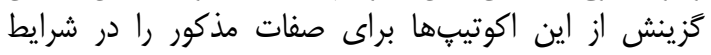
خشكى ميسر مى از ازئ د.
نتايج و بحث

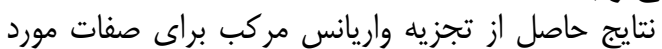

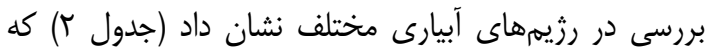

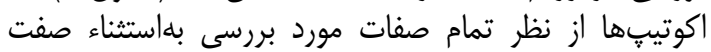


اكوتيبهاى الند، جومالو و بايقوت كمترين حساسيت را باله

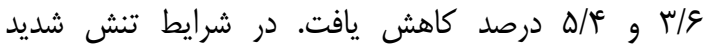

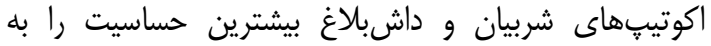

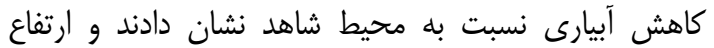

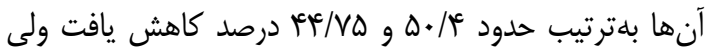

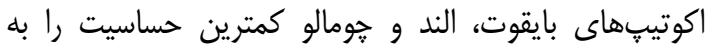

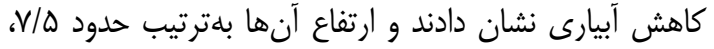

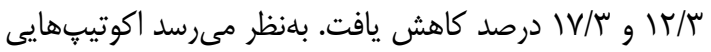

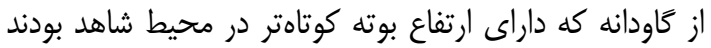

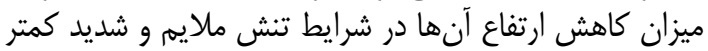

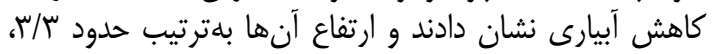

ار أفاع كياه

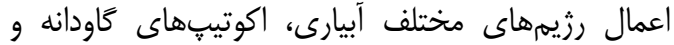

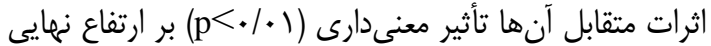

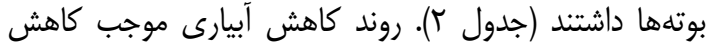

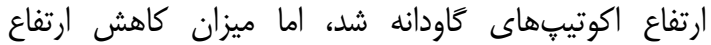

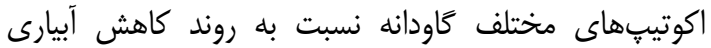

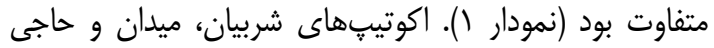

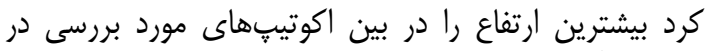

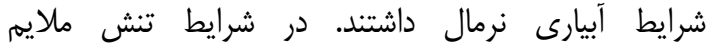

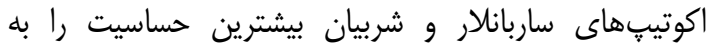

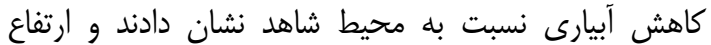

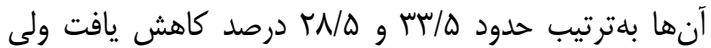

جدول r- تجزيه واريانس مركب صفات مورد مطالعه اكوتيبهاى كاودانه Table 2. Combined Analysis of variance of the studied traits of bitter vetch ecotypes

\begin{tabular}{|c|c|c|c|c|c|c|}
\hline ضريب تغييرات (\%) & خطاى r & اكوتيٍ × تنش خشكى & اكوتي" & خطاى 1 & تنش خشكى & صفت \\
\hline $1 \omega / \Delta r$ & $1 \% / q \Delta T$ & $r r / q \cdot \varphi^{*}$ & $0 \cdot 1991^{* * 3 * 3}$ & $\Delta Y / \Lambda F I$ & $\mid r \Delta \cdot / T 19^{* * *}$ & ارتفاع ساقه \\
\hline $19 / \pi$ & Q/TTQ & $N / \Lambda \Delta V^{*}$ & TI/DQ. ${ }^{*}$ & $1.19 \mathrm{VT}$ & $\mathrm{T} / \mathrm{MVV}^{\mathrm{ns}}$ & طول ريشه \\
\hline $1 Q / I f$ & $N / \Delta \cdot V$ & $|f / r| g^{*}$ & Dr/VqY & $1 / \Delta \cdot 1$ & $r q \varepsilon \Delta / M_{M}$ & تعداد غلاف در بوته \\
\hline$q / \Delta$ & $\cdot / \cdot r \cdot$ & 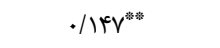 & $\cdot / T \wedge r^{* * *}$ & $.1 \cdot 18$ & T/TFY & تعداد دانه در غلاف \\
\hline$r \cdot 1 \cdot \Delta r$ & $\Delta F / T \Delta V$ & IVI/TTQ & FrN/FTV & N/TqV & $\mid V T \Lambda \cdot / F I V^{* * 0}$ & تعداد دانه در بوته \\
\hline $\mid r / \Delta \Lambda$ & $14 / 9.9$ & $r r / \cdot 9 r^{\text {ns }}$ & $r \Delta / V G r^{\mathrm{ens}}$ & $1 N / r q q^{c}$ & $r \Delta / 19 V^{n s}$ & وزن هزار دانه \\
\hline $\mid Q / \cdot 1$ & 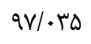 & r & $901 / 919^{* * *}$ & $\Lambda V / \cdot r V$ & 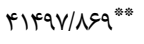 & وزن خشك اندام هوايى \\
\hline$r Y / \Delta T$ & $r \cdot / r \Delta V$ & sq/DYr & 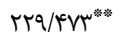 & $\Delta / \& \cdot 1$ & 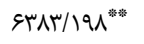 & عملكرد دانه \\
\hline
\end{tabular}

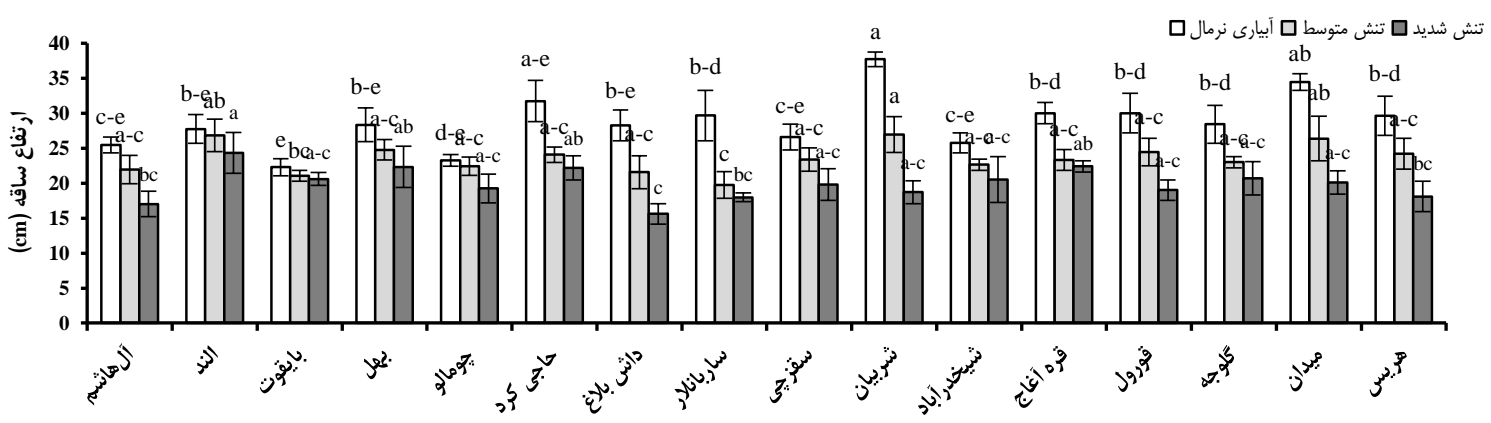

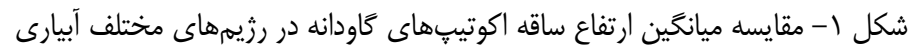

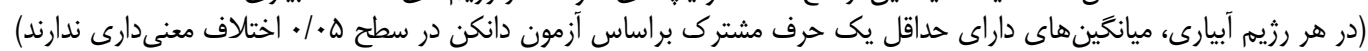

Figure 1. Means comparisons for stem height of bitter vetch ecotypes in each irrigation regime

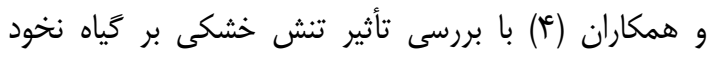
نشان دادند تنش خشكى موجب كاهش ارت ارتفاع بوته شده است طول ريشه با وجود اينكه اثر رزيمهاى متفاوت آثرات آبيارى تأثير معنى دارى بر طول ريشه نداشت، ولى اثر اكوتيبهات آنهاى كاودانه

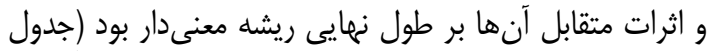

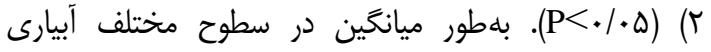

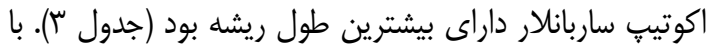

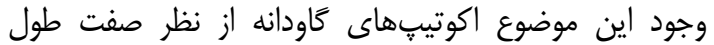

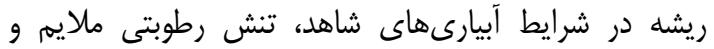

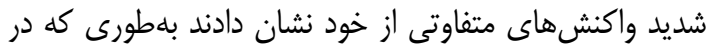
محيط شاهد اكوتيٍ ساربانالار و بايقوت بلهترتيب بيشترين وادئ و

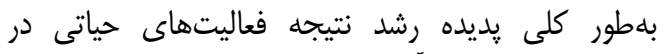

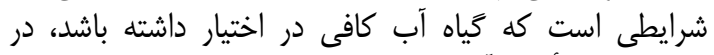

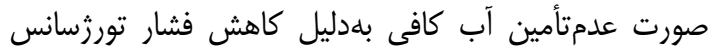

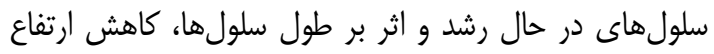

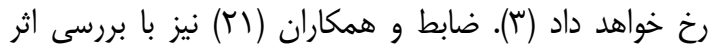

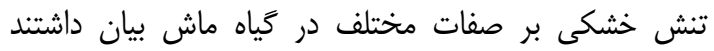

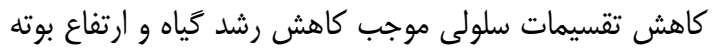

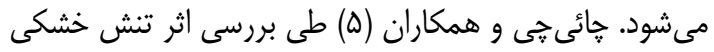

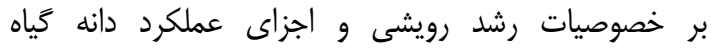

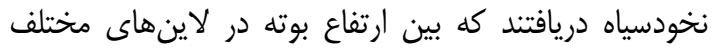

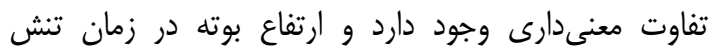

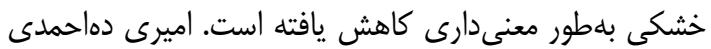


محدود و يا ضعيفتر مى كند و در اين شرايط، عوامل محيطى

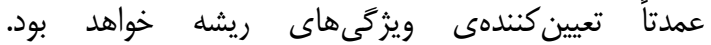

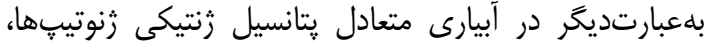

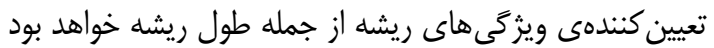

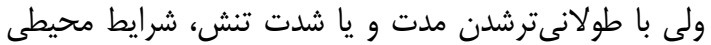

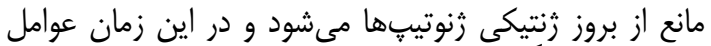

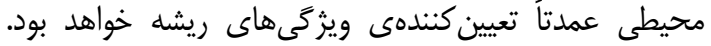

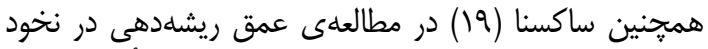

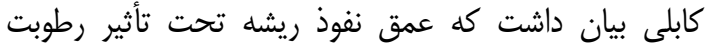
منطقه فعاليت ريشه قرار مى كيرد.

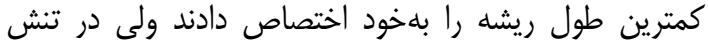

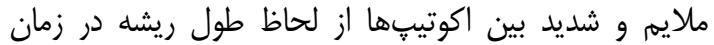

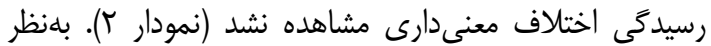

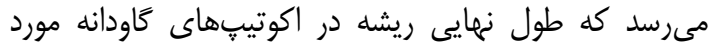

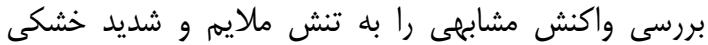

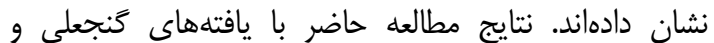
باقرى (9) مطابقت داشت. ايشان با با ارزيابى خصوصيات

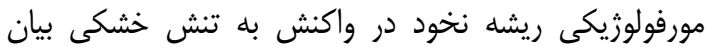

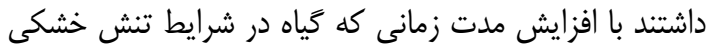

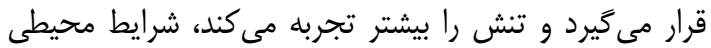

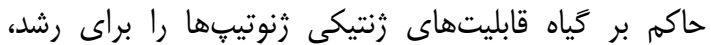

Table 3. Means comparisons of irrigation regimes and bitter vetch ecotypes for the studied trait

\begin{tabular}{|c|c|c|c|c|c|c|c|c|}
\hline $\begin{array}{c}\text { عملكرد دانه } \\
\left(\mathrm{g} / \mathrm{m}^{2}\right)\end{array}$ & هوايى ) هشى اندام & وزن هزار & تعداد دانه & تعدراد دانه & تعداد غلاف & $\begin{array}{c}\text { طول ريشه } \\
\text { (cm) }\end{array}$ & $\begin{array}{c}\text { ارتفاع ساقه } \\
\text { (cm) }\end{array}$ & اثرات اصلى \\
\hline & & & & & & & & رزيمهاى آبيارى \\
\hline$r r / q r V^{a}$ & $q r / g y)^{a}$ & $r \cdot \mid r+\varepsilon^{a}$ & $\Delta F / \cdot \Delta q^{a}$ & $r / \cdot r q q^{a}$ & $r g / r V I^{a}$ & سوqس/Fa & $r N / V \cdot l^{a}$ & آبيارى نرمال \\
\hline$r \mid e r I^{b}$ & $\Delta q / m \backslash \Lambda^{b}$ & $\Psi / \Delta V V^{a}$ & $r F / M F^{b}$ & I/AFe & $1 / N / \Lambda q^{b}$ & $|r / r \Lambda|^{a}$ & rr/ors & تنش ملايم \\
\hline \multirow[t]{2}{*}{$\mid r / \cdot r \Delta^{c}$} & $\uparrow \Psi / \wedge q \gamma^{c}$ & rN/৭q ${ }^{\mathrm{a}}$ & $r / r \Delta \Lambda^{c}$ & $1 / 99 \Delta^{c}$ & $\mid r / g V \Delta^{c}$ & $\mid f / \Delta q .^{a}$ & $19 / 9.9^{c}$ & تنش شديد \\
\hline & & & & & & & & اكوتيبهاى كَاودانه \\
\hline$I V / \Delta \cdot 9^{f}$ & $\Delta V / \& V T^{f}$ & $\Pi / 9 \Delta \omega^{\mathrm{a}}$ & $\mathrm{rq} / \mathrm{sM} \mathrm{M}^{\mathrm{f}}$ & $1 / \Delta \Delta \Lambda^{g}$ & $1 N /|g|^{d}$ & $I K / D I r^{f g}$ & $r / 4 \& q^{g}$ & آل هاشم \\
\hline$r / 1 . .$. c-e & $g q / 9 \wedge r^{c-e}$ & $r \cdot / r 19^{a}$ & $r V / V \cdot V^{c-e}$ & $1 / \Lambda \cdot r^{d-f}$ & $r \cdot / q r r^{b c}$ & lQ/Tr.bc & $r g / T \wedge V^{a-c}$ & الند \\
\hline$r \Delta / \cdot r \Lambda^{a}$ & NT/TrEa & $\Pi / \Lambda \varepsilon .^{a}$ & $\Delta r / q m \varphi^{a}$ & $1 / a \wedge m^{a-c}$ & $r g / T r r^{a}$ & $1 r / \Lambda 19^{e-g}$ & $r \mid r \cdot r^{g}$ & بايقوت \\
\hline$r \cdot 1 \cdot r r^{d-f}$ & gr/gr. ${ }^{c-f}$ & rthpera & 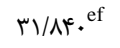 & 1/veref & $\mid V / \& \wedge \Delta^{d}$ & $I r / M \Lambda e^{b-g}$ & $r \Delta / / r \omega^{a-f}$ & بهل \\
\hline$r \cdot / \Delta \Delta r^{d-f}$ & $\Delta Q / \wedge r r^{e f}$ & $r V / V q r^{a}$ & $\left.|r|\right|^{F q T C-e}$ & $1 / a r r^{b-d}$ & $|N /| F \Lambda^{d}$ & $1 r / 9 r V^{d-g}$ & rI/Areffg & جومالو \\
\hline WNVTN & DQ/Erref & rו/v"a & $r \cdot 1 \cdot 1 *^{f}$ & $1 / \varepsilon \wedge r^{f g}$ & $I V / V q^{d}$ & $1 F / \cdot r q^{b-f}$ & $r \Delta / ৭ १ q^{a-d}$ & حاجى كرد \\
\hline$r y / \cdot 1 \varepsilon^{\mathrm{d}-\mathrm{f}}$ & $\Delta V / \Delta q \varsigma^{f}$ & $r r / \Lambda \| I^{a}$ & m/rer & I/VDFef & $|N /| \cdot \Delta^{d}$ & $|r /| \varepsilon q^{g}$ & $T / / \Lambda 10^{e-g}$ & داش بلاغ \\
\hline $19 / 1 \cdot 9^{d-f}$ & $g q / V) \Lambda^{c d}$ & $r q / r \Delta \Lambda^{a}$ & $m r / \Lambda v^{d-f}$ & $1 / \wedge \ldots{ }^{d-f}$ & $\left|N / T^{d}\right|^{d}$ & $I V / D \cdot r^{a}$ & $r r / F \& r^{d-g}$ & ساربانلار \\
\hline$r N / I I V^{b}$ & รV/৭q.cd & $r q / g r q a$ & $f \Delta / r q \Delta^{b}$ & $r / M \Lambda^{\mathrm{a}}$ & $r y / \wedge r^{b}$ & $\mid f / .9 \Delta^{b-f}$ & سم/rc-g & سقزجى \\
\hline$r \cdot / \Lambda \Delta \mu^{d-f}$ & qאוא & $r r / \Lambda r \Lambda^{a}$ & $r \Delta / F^{\leftarrow} \vee \omega^{c-f}$ & $1 / \Lambda \mid r^{d-f}$ & $|N / V \Lambda|^{b-d}$ & $10 / \mathbb{F r a b}^{b}$ & $r V / V V r^{a}$ & شربيان \\
\hline$r r / \cdot q r^{c-} e$ & $\Delta V / \Delta r V^{f}$ & ri|ra & & $1 / \wedge 9 \Delta^{c-e}$ & $\mid N / \varepsilon \Delta V^{c d}$ & $|r / F \Delta|^{c-g}$ & $r r / q \Delta \varepsilon^{c-g}$ & شيخدرآباد \\
\hline rळ/१q. bc & $V \Lambda / F_{Y} F^{a b}$ & $r \cdot / q v v^{a}$ & $r \cdot / \wedge \ldots b c$ & $r / \cdot q \cdot{ }^{a}$ & $19 / \cdot 9 v^{b-d}$ & $\mid f / F r \varepsilon^{b-e}$ & $r \Delta / r r \omega^{a-e}$ & قره آغاج \\
\hline rm/אred & $\vee / / q^{b c}$ & $r \cdot / \kappa \wedge r^{a}$ & $r \Delta / \xi \cdot \Lambda^{c-f}$ & $1 / \wedge \vee \xi^{c-e}$ & N/Dq. ${ }^{\mathrm{cd}}$ & $1 f / \Lambda v^{b-f}$ & $r r / F V \Lambda^{a-g}$ & قورول \\
\hline$r / \Delta \Delta)^{c d}$ & $\left.9 \Delta / q)^{c}\right)^{c-e}$ & $r \cdot / 9 \Lambda \cdot{ }^{a}$ & rN/Vז山 ${ }^{c d}$ & $r / \cdot \kappa^{a a b}$ & $19 / 1 r^{b-d}$ & $\mid r / \cdot r f^{d-g}$ & $r \xi / \cdot r \cdot{ }^{b-g}$ & كلوجه \\
\hline$r \cdot \mid \Delta r r^{d-f}$ & $\mathrm{gV} / \mathrm{IV} \cdot{ }^{\mathrm{c}-\mathrm{e}}$ & $r \cdot|q r|^{a}$ & $r F / V^{m} v^{d-f}$ & $1 / v q r^{d-f}$ & $W / V \wedge V^{c-d}$ & $1 f / q^{c} v^{b-d}$ & $r \varepsilon / q \vee \varepsilon^{a b}$ & ميدان \\
\hline W/eFef & $\Delta Q / \mathcal{G})^{\text {ef }}$ & $r V / q r \Delta^{a}$ & $r r / \Lambda r \omega^{d-f}$ & $1 / v \cdot v^{f}$ & WN/KrT & $10 / 119^{b c}$ & $r \mu / Q q .{ }^{b-g}$ & هريس \\
\hline
\end{tabular}

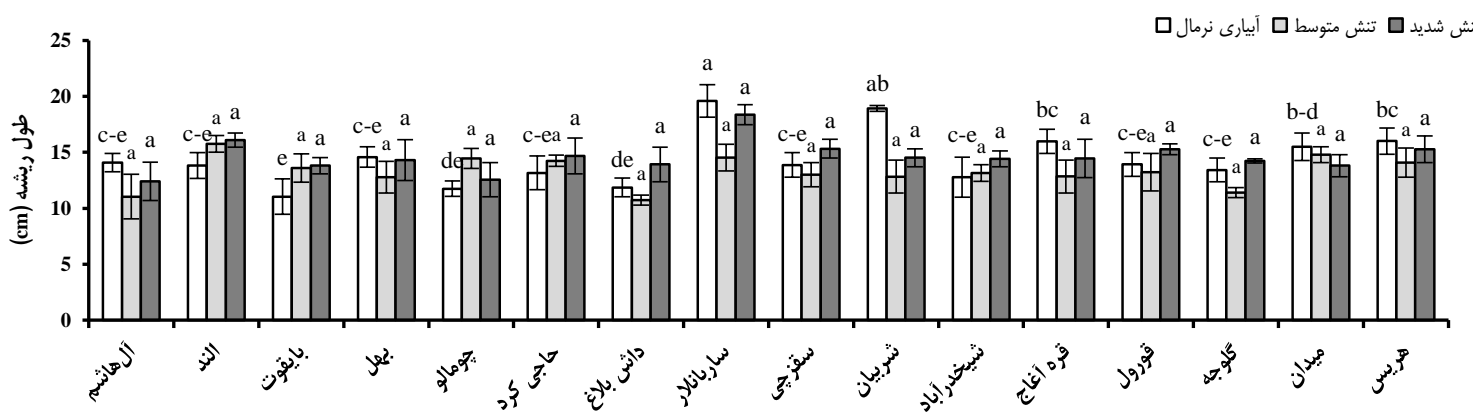

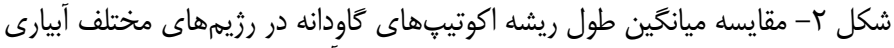

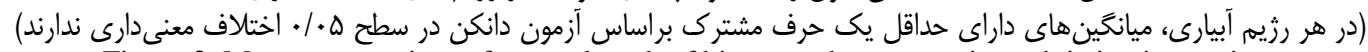

Figure 2. Means comparisons for root length of bitter vetch ecotypes in each irrigation regime 
درصد كاهش نسبت به شاهد در آبيارى تنش ملايم و

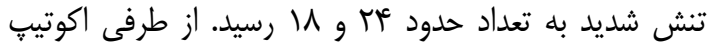

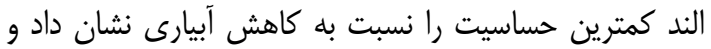

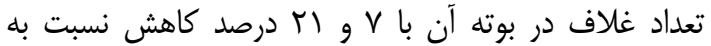

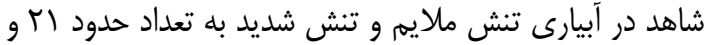

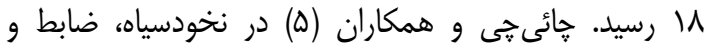

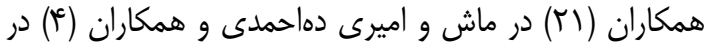

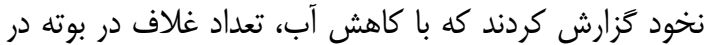

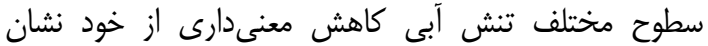
مى دهند.

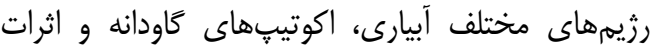

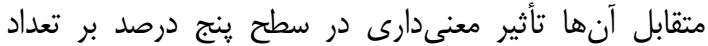

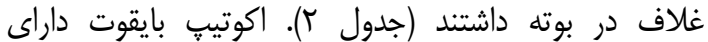

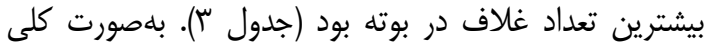

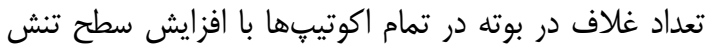

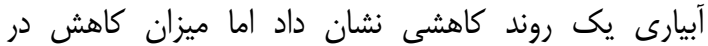

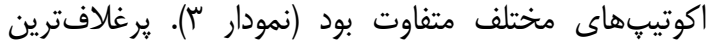

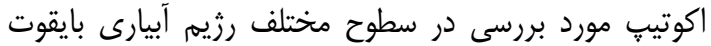

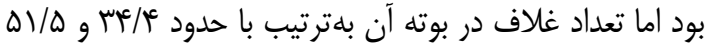

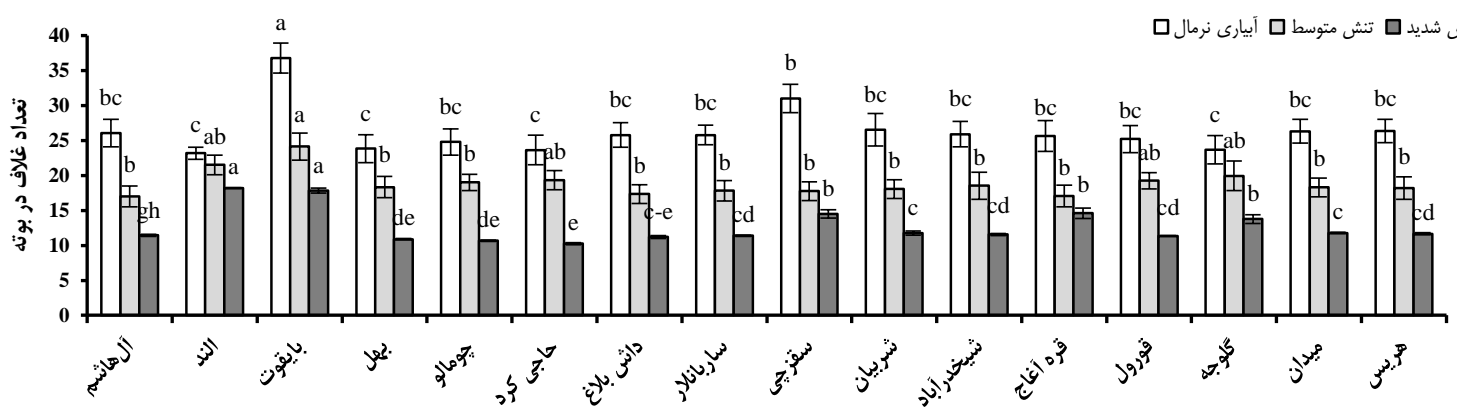

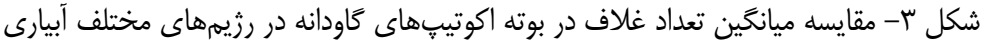

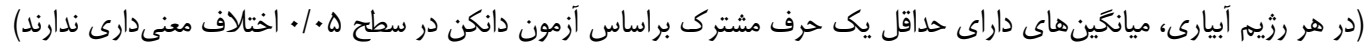

Figure 3. Means comparisons for pod number per plant of bitter vetch ecotypes in each irrigation regime

ساربانلار، قرهآغاج و سقزجى، در تنش ملايهم اكوتيڤهاى

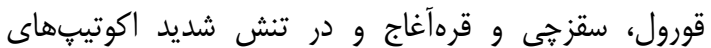

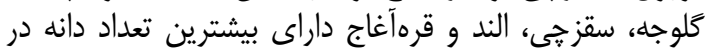

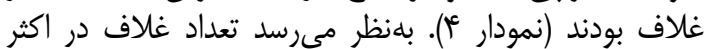

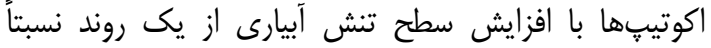

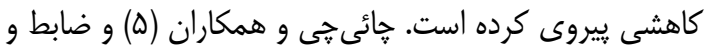

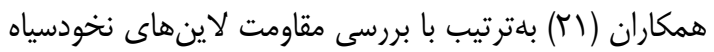

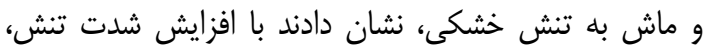

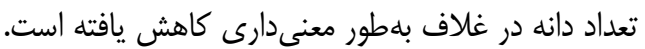

تعداد دانه در غلاف

اثر رزيمهاى متفاوت آبيارى، اكوتيبهاى داى كاودانه و اثرات

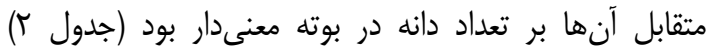

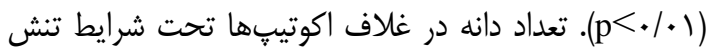

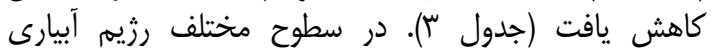

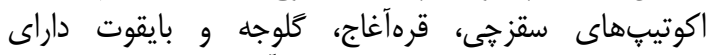

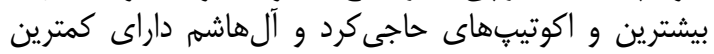

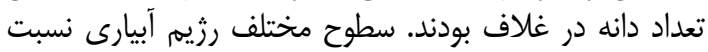

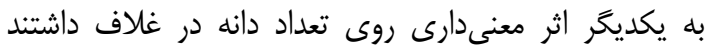

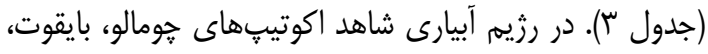

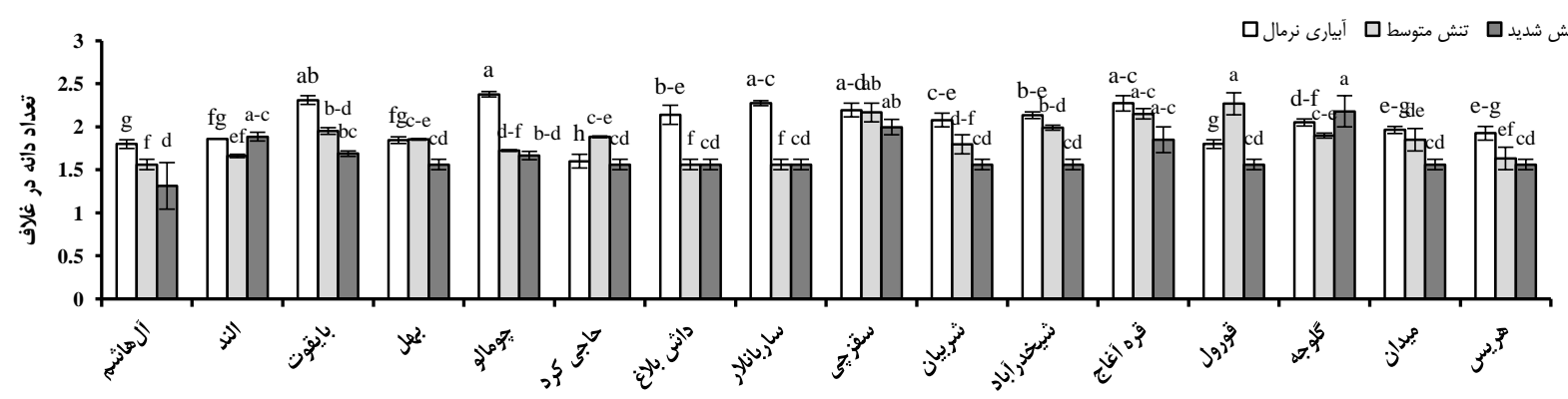

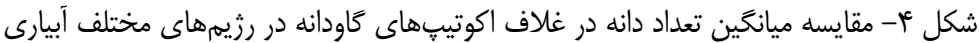

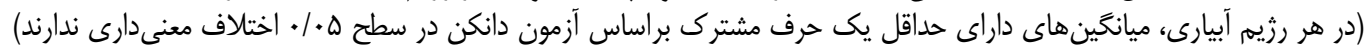

Figure 4. Means comparisons for seed number per pod of bitter vetch ecotypes in each irrigation regime 
كاهش تعداد دانه در بوته كردد (1)). در شرايط آبيارى شاهد:

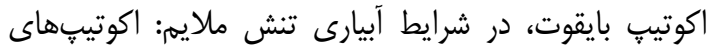

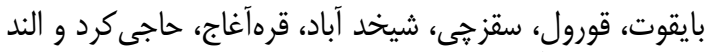

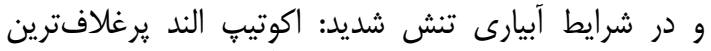

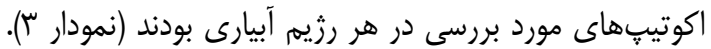

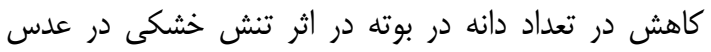

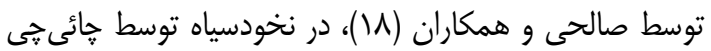

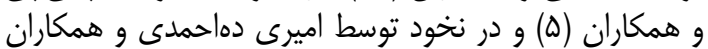

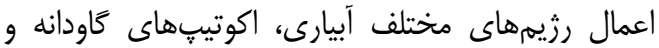

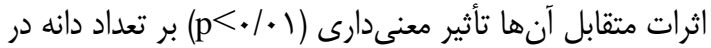

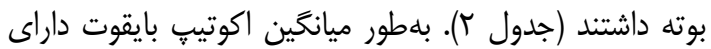

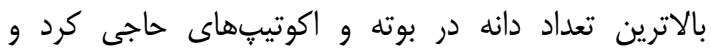

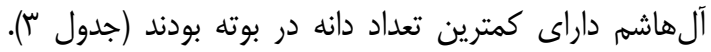

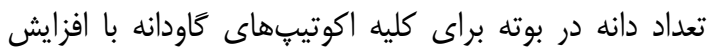

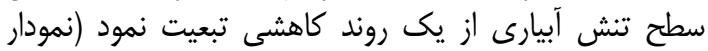

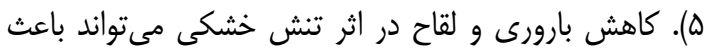

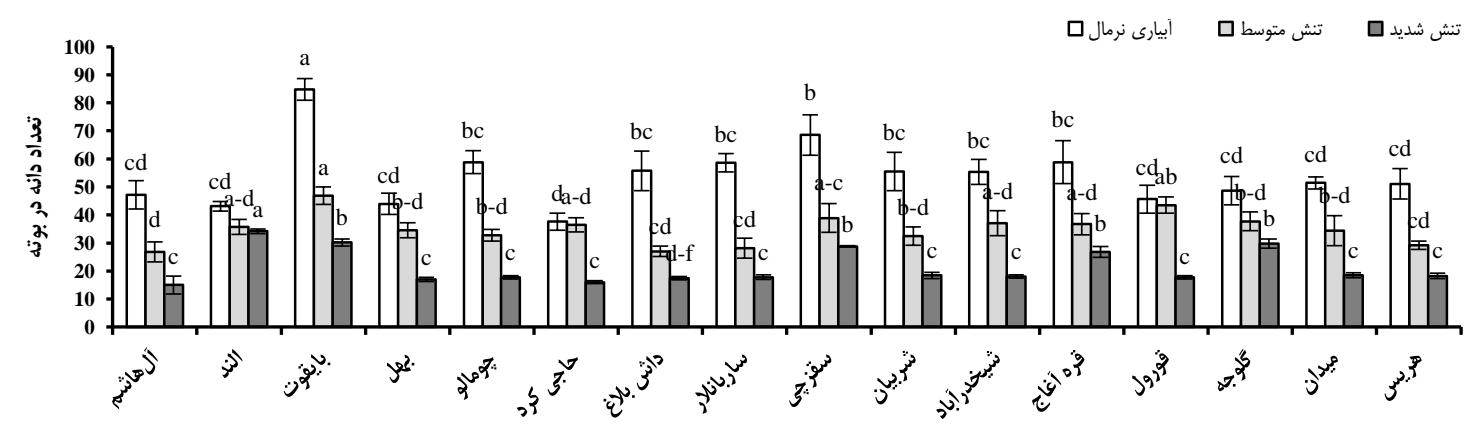

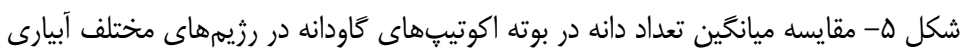

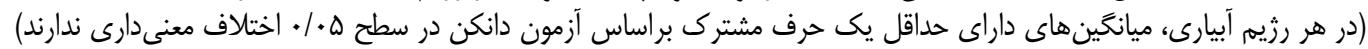

Figure 5. Means comparisons for seed number per plant of bitter vetch ecotypes in each irrigation regime

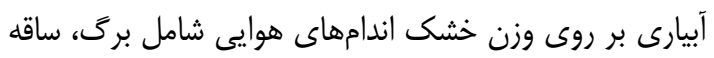

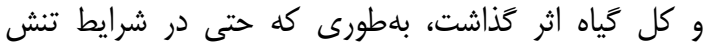

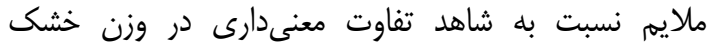

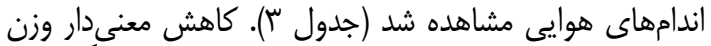

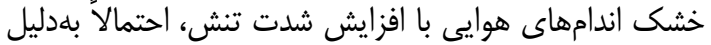

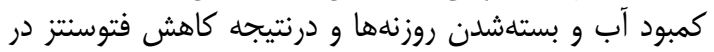

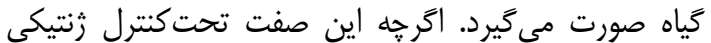

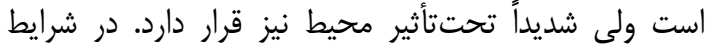

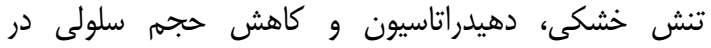

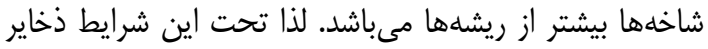

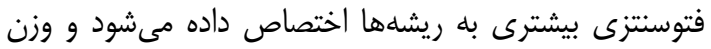

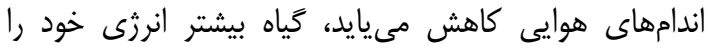

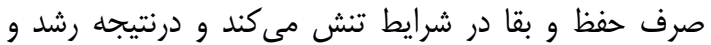

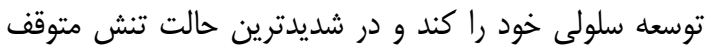

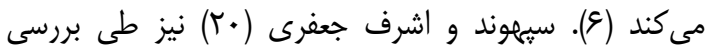

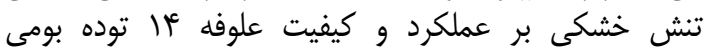

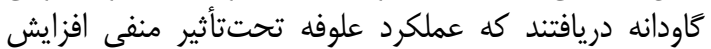

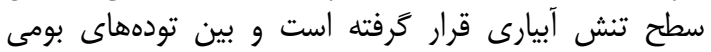

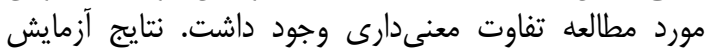

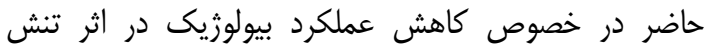

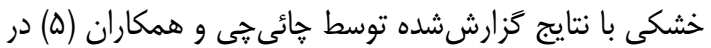

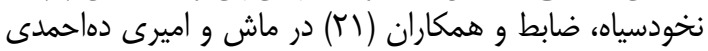
و همكاران (ז) در نخود تطابق دارد.
وزن هزاردانه

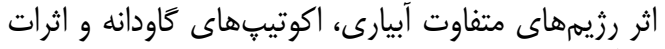

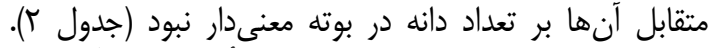

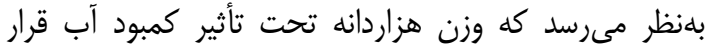

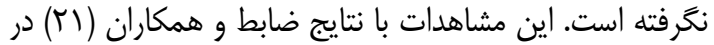

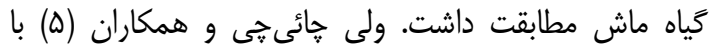

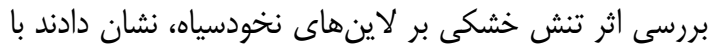

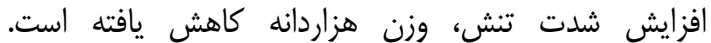

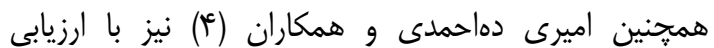

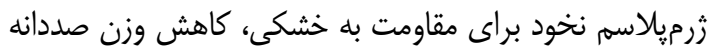

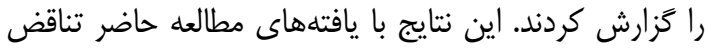

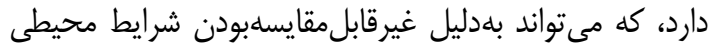

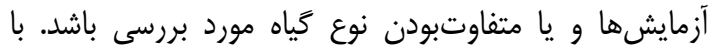

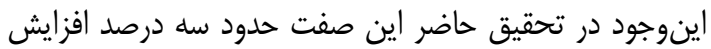

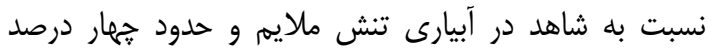

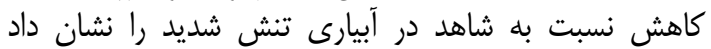

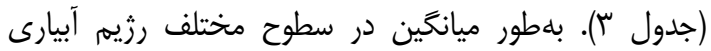

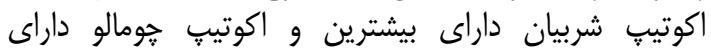
كمترين وزن هزار دانه بودند (جدول ساب ). وزن خشك اندام هوايى

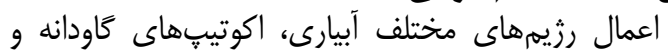

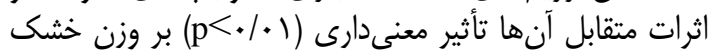

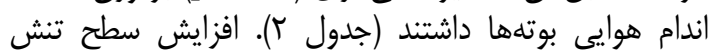




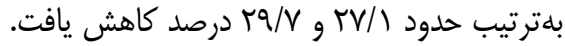

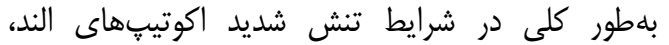

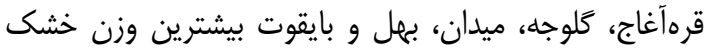

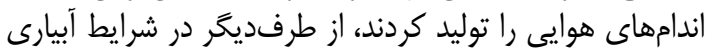

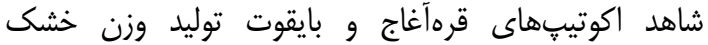

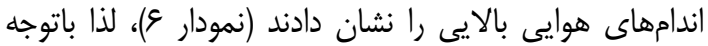

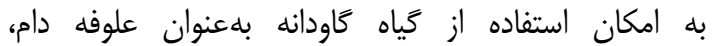

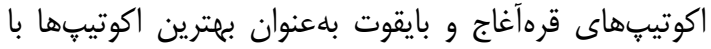

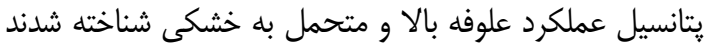

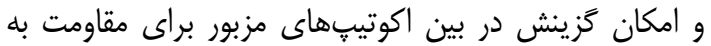

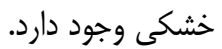

در شرايط آبيارى تنش ملايم اكوتيِهاى داشبلاغ،

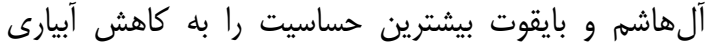

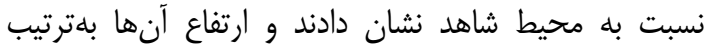

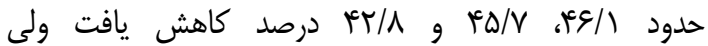

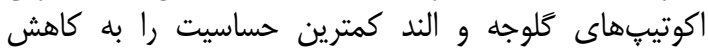

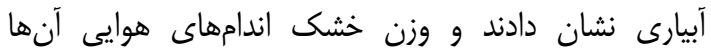

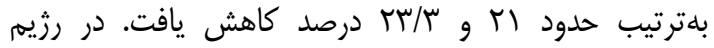

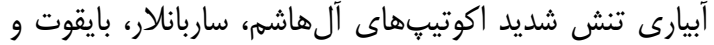

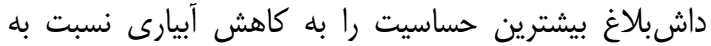

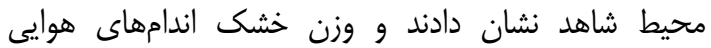

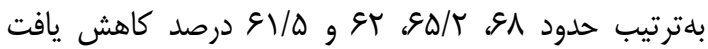

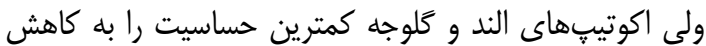

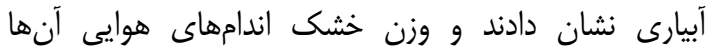

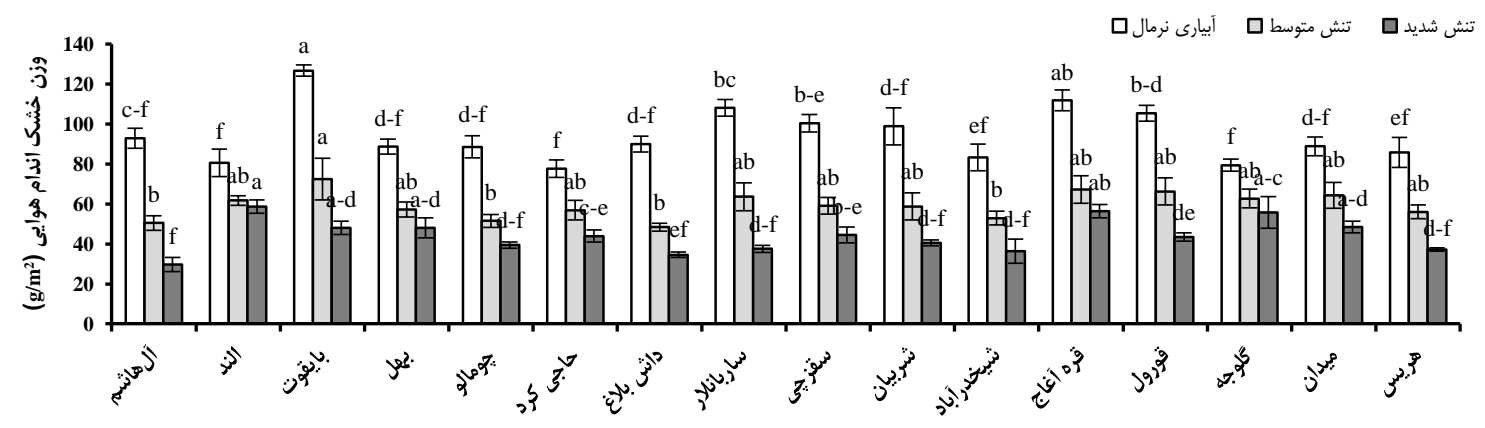

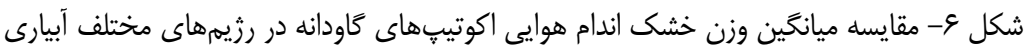

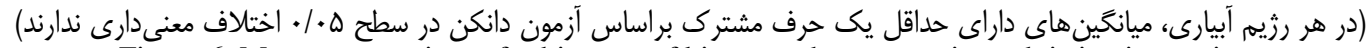

Figure 6. Means comparisons for biomass of bitter vetch ecotypes in each irrigation regime

در شرايط تنش خشكى است (•l(). واكنش اكوتيبهاى

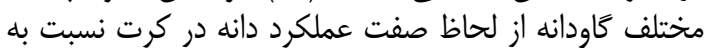

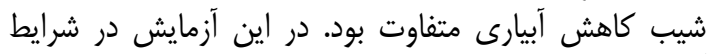

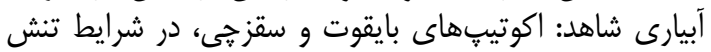

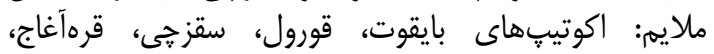

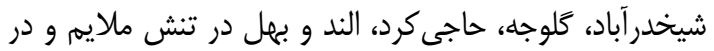

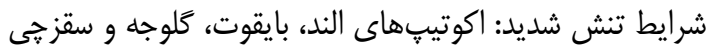

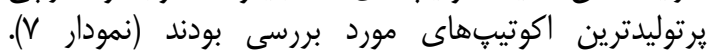

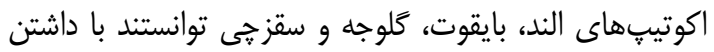

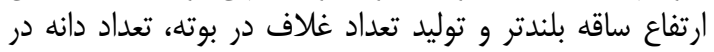

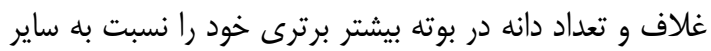

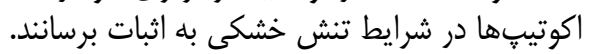

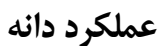

اثر رزيمهاى متفاوت آبيارى، اكوتيِهاى كاوداودانه و اثرات

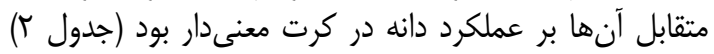

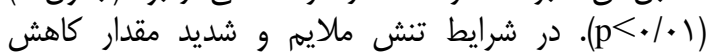

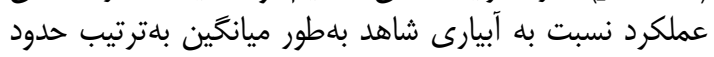

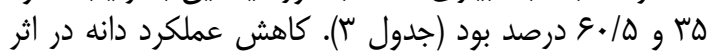

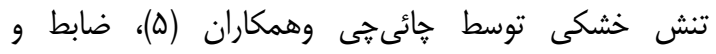

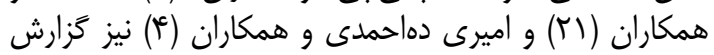

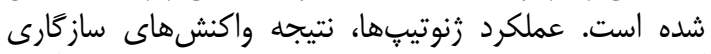

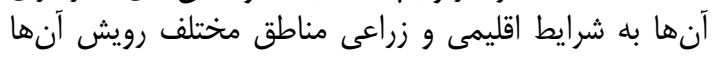

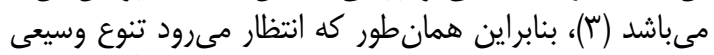

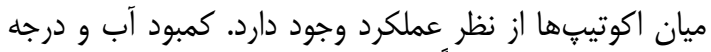

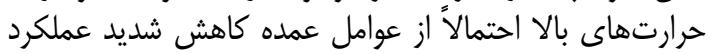




$$
60 \text { تنش شديده }
$$

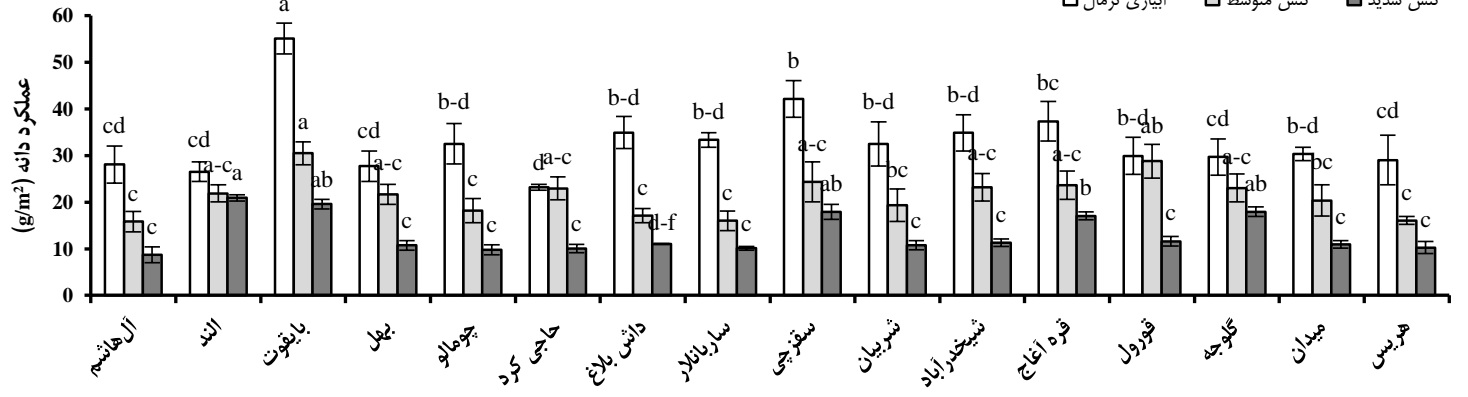

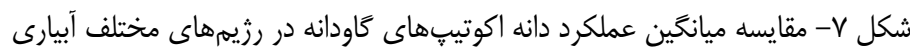

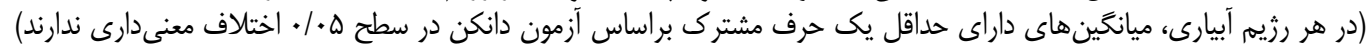

Figure 7. Means comparisons for grain yield of bitter vetch ecotypes in each irrigation regime

نتايج حاصل از تحقيق حاضر نشان داد تنوع زنتيكى در

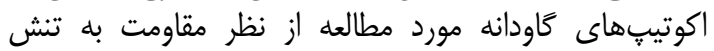

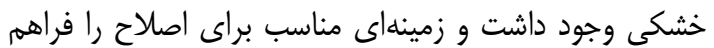

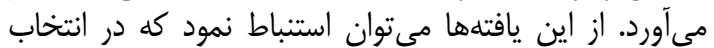

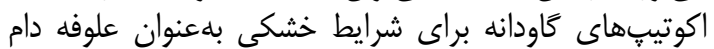

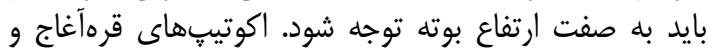

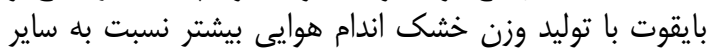

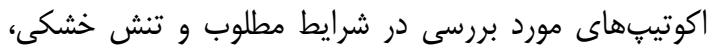

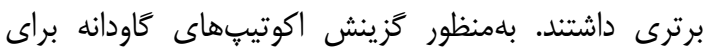

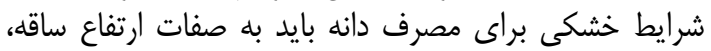

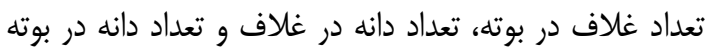

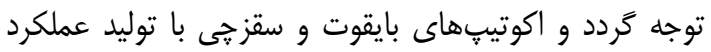

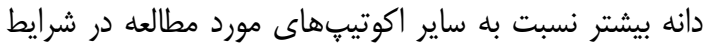

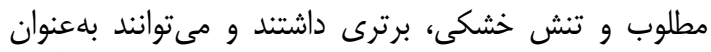

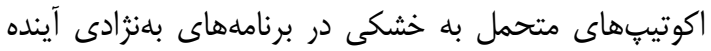
مورد استفاده قرار گيرند.
همانطور كه كفته شد اين اكوتيِها مقادير بالاترى از

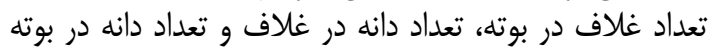

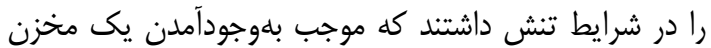

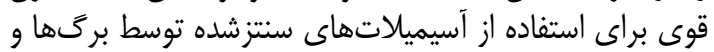

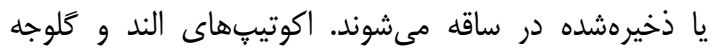

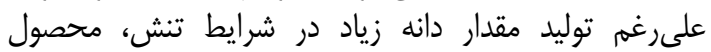

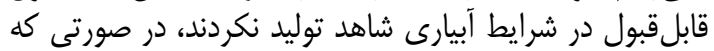

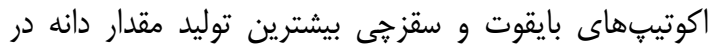

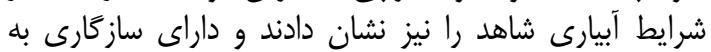

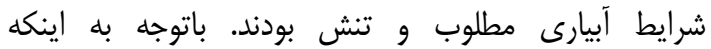

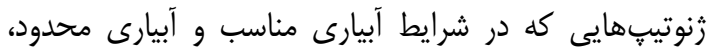

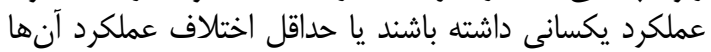

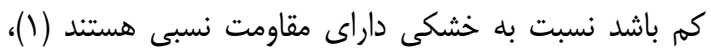

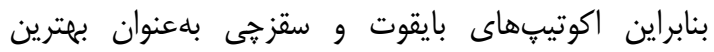

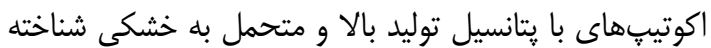

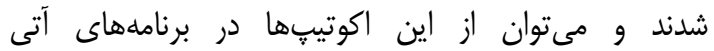

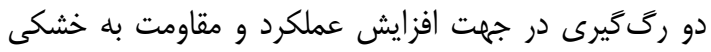

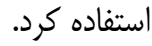

1. Abd Mishani, C and J. Jafari Shabestari. 1988. Evaluation of wheat cultivars for drought resistc Iranian Journal of Agriculture Science, 19(1-2): 37-43 (In Persian).

2. Abdullah, A.Y., M.M. Muwalla, R.I. Qudsieh and H.H. Titi. 2010. Effect of bitter vetch (Vicia ervilia) seeds as a replacement protein source of soybean meal on performance and carcass characteristics of finishing Awassi lambs. Tropical Animal Health and Production, 42: 293-300.

3. Ahmadi, A. and D.A. Baker. 2000. Stomatal and nonstomatal limitations of photosynthesis under water stress conditions in wheat plant. Iranian Journal of Agriculture Science, 31(4): 813-825 (In Persian).

4. Amiri Deh Ahmadi, S.R., M. Parsa and A. Gangali. 2011. The effects of drought stress at different phenological stages on morphological traits and yield components of chickpea (Cicer arietinum L.) under greenhouse conditions. Iranian Journal of Field Crops Research, 8(1): 157-166 (In Persian).

5. Chaichi, M.R., M. Rostamza and K. Sadat Esmaeilan. 2004. Tolerance evaluation of chichpea accessions to drought stress under different irrigation systems during generative growth stage. Journal of Agricultural Science and Natural Resource, 10(4): 55-64 (In Persian).

6. Edalati Fard, L., S. Galeshi, A. Soltani and F. Akram Ghaderi. 2007. The role of morphophysiological traits in drought tolerance of cotton genotypes. Journal of Agricultural Science and Natural Resource, 13(2) (In Persian).

7. Fakhre Vaezi, A. 2013. Evaluation of yield stability and adaptability of vetch varieties (Vicia ervilia) in dryland conditions. Journal of Agricultural Science and Natural Resource, 15: 51-55 (In Persian). 
8. Fathi Rezaee, V., M. Valizadeh, Kh. Alizadeh and S. Zehtab Salmasi. 2006. Evaluation of bitter vetch lines under irrigated and rainfed conditions. Journal of Sustainable Agrictulture and Production, 2/20(1): 23-37 (In Persian).

9. Ganjali, A. and A. Bagheri. 2011. Evaluation of morphological characteristics of root chickpea (Cicer arietinum L.) in response to drought stress. Iranian Journal of Pulses Research, 1(2): 101-110 (In Persian).

10. Ganjali, A., A. Bagheri and H. Porsa. 2010. Evaluation of chickpea (Cicer arietinum L.) germplasm for drought resistance. Iranian Journal of Field Crops Research, 7(1): 183-194 (In Persian).

11. Haddad, S.G. 2006. Bitter vetch grains as a substitute for soybean meal for growing lambs-livestock. Science, 99: 221-225.

12. Huang, B. 2000. Role of root morphological and physiological characteristics in drought resistance of plant. In: R.E. Wilkinson (ed) Plant Environmental Interaction, Marcel Dekker Inc, New York, USA, 39-40 pp.

13. Manavalan, L.P., S.K. Gutikonda, L.S.P. Tram and H.T. Nguyen. 2009. Physiological and molecular approaches to improve drought resistance in soyebean. Plant Cell Physiology, 50: 1260-1276.

14. Rastegar, M.A. 2005. Forage crops production. Brrahmand Publisher, Tehran, Iran, (In Persian).

15. Razmazar, V., N.M. Torbatinejad, J. Seifdavati and S. Hassani. 2012. Evaluation of chemical characteristics, rumen fermentation and digestibility of Vicia sativa, Lathyrus sativus and Vicia ervilia grain by in vitro methods. Journal of Animal Science Researches, 22(2): 107-119 (In Persian).

16. Sadeghi, G.H., J. Pourreza, A. Samei and H. Rahmani. 2009. Chemical composition and some antinutrient content of raw and processed bitter vetch (Vicia ervilia) seed for use as feeding stuff in poultry diet. Tropical Animal Health and Production, 41: 85-93.

17. Sahhafi, S.R. and B. Maleki Zanjani. 2016. Analysis of geneticvariation for morphological and agronomictraits in bitter vetch landraces. The $2^{\text {nd }}$ international and $14^{\text {th }}$ National Iranian Crop Science Congress, Rasht, Iran, 1-5 (In Persian).

18. Salehi, M., A. Haghnazari, F. Shekari and A. Faramarzi. 2006. Factor analysis of yield, yield components and some effective characters for drought resistance in lentil (Lens culinaris Medik). Journal of Agroecology, 2(4): 71-86 (In Persian).

19. Saxena, M.P. 2003. Manangement of agricultural drought: Agronomicand geneticoptions. Science Publishers, INC, India.

20. Sepahvand, A. and A. Ashraf Jafari. 2014. Study for yield and quality traits in 14 domesticpopulations of bitter vetch (Vicia ervilia) in optimum and dry condition in Khoramabad, Iran. Agronomy Journal, 102: 20-30 (In Persian).

21. Zabet, M., A.H. Hosein Zade, A. Ahmadi and F. Khialparast. 2003. Effect of water stress on different traits and determination of the best water stress index in mung bean (Vigna radiate). Iranian Journal of Agriculture Science, 34 (4): 889-898 (In Persian). 


\title{
Evaluation of Drought Resistance of Bitter Vetch Ecotypes in Rafsanjan, Iran
}

\section{Mohadeseh Ghanipour Govarki ${ }^{1}$, Seyyed Rasoul Sahhafi ${ }^{2}$, Aliakbar Mohammadi Mirik ${ }^{3}$ and Asghar Rahimi ${ }^{4}$}

1- M.Sc. Student of Vali-e-Asr University of Rafsanjan

2- Assistant Professor of Vali-e-Asr University of Rafsanjan, (Corresponding author: s.r.sahhafi@ vru.ac.ir)

3 and 4- Assistant Professor and Associate Professor of Vali-e-Asr University of Rafsanjan Received: January 11, $2019 \quad$ Accepted: May 26, 2019

\begin{abstract}
The aim of this research was to assessment of drought tolerance in bitter vetch (Vicia ervilia L.) ecotypes. For this purpose, 16 ecotypes collected from four provinces (East Azerbaijan, West Azerbaijan, Ardabil and Zanjan) of Iran, were evaluated using randomized complete block design with four replications in three separate experiments (60,90 and $120 \mathrm{~mm}$ water evaporation from A pan) during 2017-2018 growing season in the Research Field of Faculty of Agriculture at Vali-e-Asr University of Rafsanjan. The result of combined analysis of variance showed there were significant differences at $1 \%$ level between the ecotypes for all traits except thousand kernel weight in their reaction to drought stress. Drought stress caused decrease in stem height, root length, pod number per plant, seed number per pod, seed number per plant, biomass and grain yield, significantly. Ecotype Byghout produced the most biomass (by an average of $82.345 \mathrm{~g} / \mathrm{m}^{2}$ ) and grain yield (by an average of $35.038 \mathrm{~g} / \mathrm{m}^{2}$ ) over all irrigation levels. Means comparisons showed that ecotypes Ghareaghaj and Byghout (respectively) was more stable in biomass production as well as ecotypes Byghout and Saghazchi (respectively) was more stable in grain yield production across all irrigation levels. In general, these ecotypes that were less affected by drought stress can be used as promising genotypes in future breeding programs with the objective of enhanced drought resistance.
\end{abstract}

Keywords: Bitter Vetch, Drought Stress, Grain Yield, Resistance 